\title{
Shocks in fragile matter
}

ص Vincenzo Vitelli ${ }^{1}$, Martin van Hecke ${ }^{2}$ - DOI: 10.1051/epn/2012606

I ${ }^{1}$ Instituut Lorentz for Theoretical Physics - Leiden University - P.O. Box 9506 - NL-2300 RA Leiden - The Netherlands.

- ${ }^{2}$ Kamerlingh Onnes Lab, Leiden University - P.O. Box 9504 - NL-2300 RA Leiden - The Netherlands.

In ordinary solids, only the most violent impacts lead to acoustic shocks. Soft materials, such as granular media, are different. They can be prepared in a state of vanishing rigidity, where even the tiniest perturbations create shock waves. Such materials are not just soft, they are fragile. 


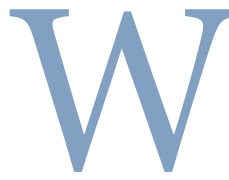

hen a jet breaks through the sound speed barrier, it releases a great amount of energy in the form of non-linear sound waves often visible as a condensation cloud of the cooling air (see introductory illustration). This spectacular phenomenon, reminiscent of an explosion, is called a sonic boom and is a striking example of an acoustic shock. What about acoustic shocks propagating through a solid?

Sound speeds in metals are much higher than in air. A bullet would need to move faster than $6000 \mathrm{~m} / \mathrm{s}$ into a solid block of steel for it to create an acoustic shock (see Fig. 1a). This is clearly a situation of scarce relevance to

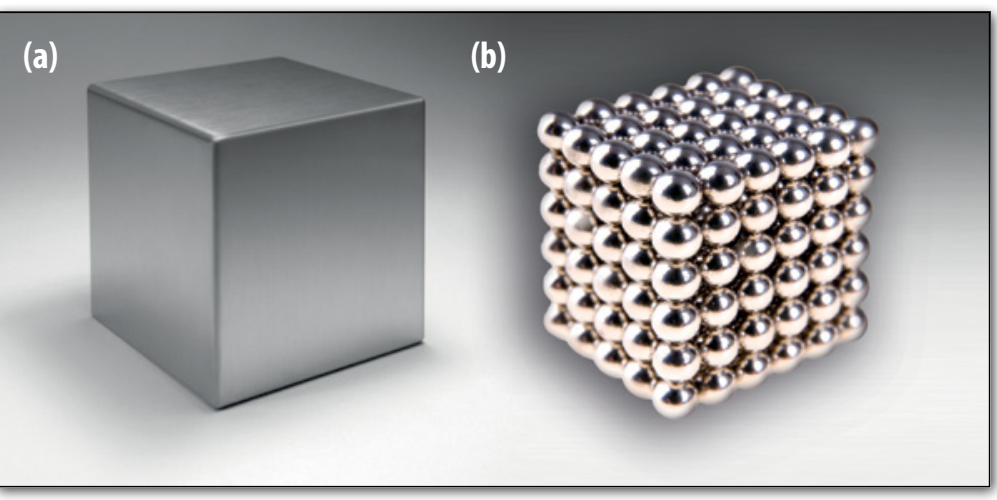

everyday life. However, if, instead of a solid block, one considers a loose packing of steel balls (see Fig. 1b), a distinct physical property emerges: fragility. Such loose packings of balls or other particles are called granular media (sand, cereal, sugar). Granular media can display vanishingly small sound speeds, even if the particles are made of steel. When the sound speed is lowered to zero, linear sounds waves become irrelevant: every applied perturbation, no matter how tiny and slow, propagates supersonically, in the form of shock waves. How does this happen?

\section{The Sonic Vacuum}

Individually, steel balls are neither soft nor fragile. Collectively though, granular packings are fragile and soft. The root cause of this lies in the non-linear, Hertzian interaction law, which governs the elastic deformations of spheres in contact (see Box 1). According to this law, when you push two elastic balls together, the deformation $\delta$ varies with the force $f$ as $f \sim \delta^{3 / 2}$. If the contact law would have been linear, as you might have expected, doubling the force would double the deformation, but Hertzian contacts get stronger the more you compress. And, conversely, they get weaker and weaker the less you compress. More precisely, when many such balls are arranged along a chain, the effective spring constant, $k_{\text {eff }}$, is not really a constant anymore! Instead $k_{\text {eff }} \sim \mathrm{d} f / \mathrm{d} \delta \sim \delta^{1 / 2} \sim \mathrm{f}^{1 / 3}$ can be tuned by varying the compression $\delta$, and vanishes when the confining force goes to zero. This has a crucial effect on the speed of sound of the chain: $c \sim k_{\mathrm{eff}}{ }^{1 / 2}$. So, while the sound speed within the particles, $v_{\mathrm{g}}$, is very large and independent of compression, the weak contacts lead to a vanishing collective sound speed when the overlap $\delta$ tends to zero. Such a state of vanishing rigidity and sound speed is called the sonic vacuum [1].

The sonic vacuum state of a Hertzian chain reminds us of the Newton's cradle, a toy composed of pendulum balls just touching so that $\delta=0$, see Fig. 2 . When the leftmost ball strikes its neighbour with speed $u_{\mathrm{p}}$, a propagating pulse of mechanical energy is generated, despite the vanishing sound speed. But, if the sound speed is zero, what excitations can propagate through this fragile mechanical system? And what sets their speed $v_{s}$ ? Nesterenko first answered these questions in his pioneering investigation $\triangle$ Fig. 1: (a) A solid block of steel has a sound speed of $6 \mathrm{~km} / \mathrm{s}$, while a granular material, such as packings of steel balls (b), may exhibit a vanishing sound speed. $\triangleleft A$ jet breaking through the sound barrier produces a shock wave. made visible by a condensation cloud. (C) istockPhoto 
When two macroscopic objects are brought into contact an elastic deformation $\delta$ is generated, as illustrated in figure. If a ball of radius $R$ is squeezed against a flat wall, it is compressed in the longitudinal direction and expands in the transverse direction. Simple geometric considerations reveal that the radius of the area of contact is approximately $(R \delta)^{1 / 2}$ (see figure). That means that, in the contact area, the deformation (or strain) $Y$ can be estimated by dividing the longitudinal deformation $\delta$ by this radius: $\gamma=\delta /(R \delta)^{1 / 2} \sim \delta^{1 / 2}$. According to linear elasticity, the stress $\sigma$ is proportional to the strain $\gamma$ so that $\sigma \sim \delta^{1 / 2}$. The corresponding force $f$ is obtained by multiplying the stress by the area of contact, which is proportional to $\mathrm{R} \delta$, see the dashed circle in the Figure. The Hertzian law of interaction then follows: $f \sim \delta^{3 / 2}$, where only the dependence on $\delta$ has been tracked explicitly. Note that despite the linear stress-strain relation for the balls material, their interaction force is non-linear.

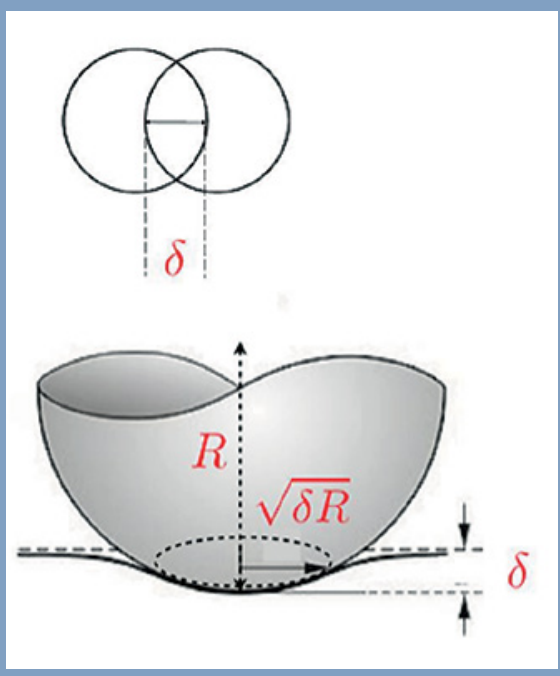

of one-dimensional granular chains [1].He demonstrated that non-linear sound propagates with speed $v_{\mathrm{s}} \sim u_{\mathrm{p}}{ }^{1 / 5}$ in the form of solitary or shock waves - note that $v_{s}$ is larger than the sound speed in the chain $c$ but lower than the speed of sound $v_{\mathrm{g}}$ within each ball. Recently, these insights have inspired technological advances ranging from tunable acoustic diodes to non-linear acoustic lenses [2].

\section{Shocks in Sand}

However, much remains to be understood about common granular materials such as sand. The confining pressure

$\checkmark$ Fig. 2:

Newtons's cradle: a sonic vacuum. () iStockPhoto

Dig. 3: Simulations reveal supersonic

shock waves travelling at a speed $v_{s}$ in response to piston compression of a model granular medium with constant speed $u_{\mathrm{p}}$.
$P$ is perhaps the most accessible experimental parameter, and it controls the average grain-overlap $\delta$. As the confining pressure is lowered to zero, the sound speed vanishes [3]. However, granular packings are not simply one-dimensional and they are clearly amorphous. Will disorder destroy the Nesterenko solitons?

We have simulated the response of two-dimensional amorphous packings of Hertzian particles to the impact of a rapidly moving piston [4], see Fig. 3. The piston compression leads to the formation of a front that separates two states: behind the front we find a compressed region in which the particles are moving on average with the piston speed $u_{\mathrm{p}}$, and ahead of the front we find an uncompressed region where the particles are at rest. Despite the strong disorder in the packings, the resulting fronts are smooth and stable, see Fig. 3. One finds two distinct types of fronts, depending on the initial pressure of the packing, and the speed of the piston. For large pressure and small piston speeds, the front speed is simply the linear sound speed, which varies with pressure but is independent of the piston speed. In contrast, for large piston speeds or small pressures, one finds more rapid shock waves, whose velocity is set by the piston speed only - much like the Nesterenko solitons (see box 2).

\section{Extreme Mechanics of Fragile Matter}

How general are such phenomena? The vanishing of the rigidity and sound speed of granular media for small pressures is just one example of fragile materials arise. For example, network glasses such as chalcogenides are made of covalent bonds (see Fig. 5). Yet, they can still be fragile if the average valence falls below a certain threshold [5]. In this case, the energy cost of microscopically
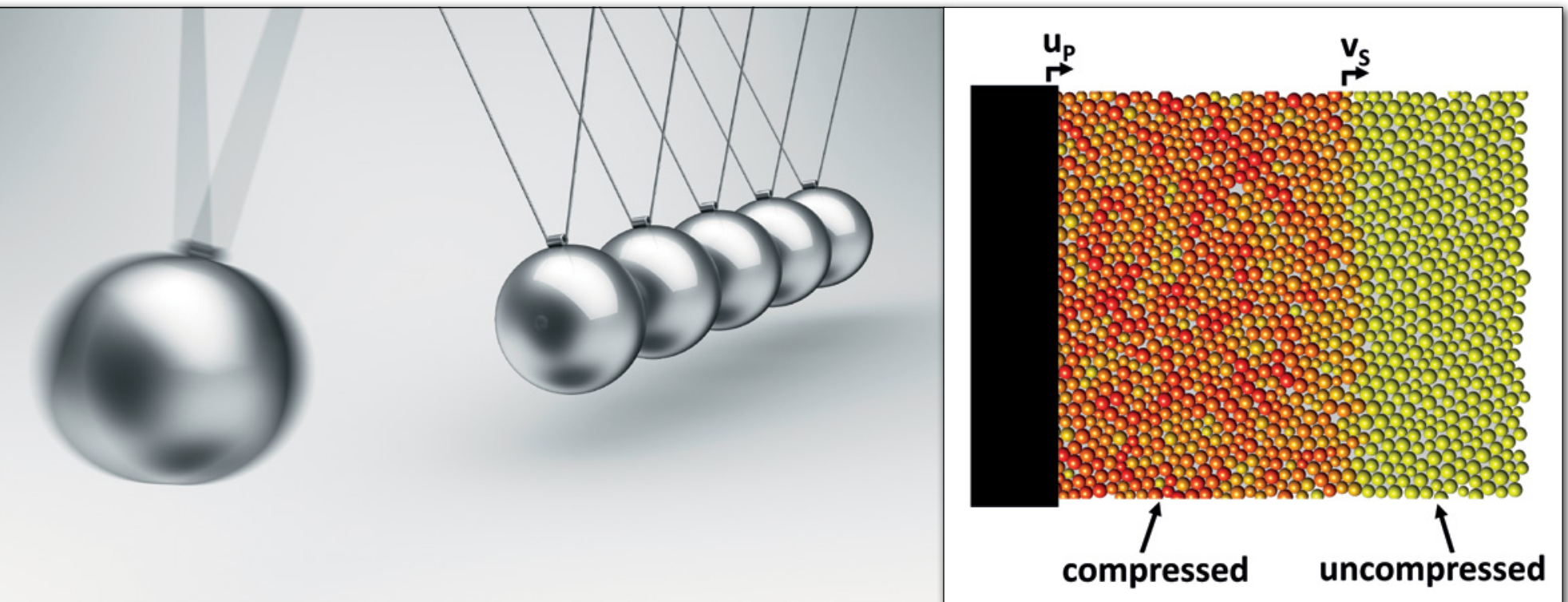
deforming a bond is harmonic: the origin of fragility cannot be traced to non-linear interatomic forces. This global sonic vacuum state is generated by the weak connectivity of the network. It is the network topology that matters, not the strength of the individual bonds.

Similarly, shock waves are just one example of the intrinsically nonlinear phenomena that arise when soft materials become fragile. Other examples include nonlinear elasticity and nonlinear flow behaviour [6-7].

A common theme thus emerges. First, a wide class of materials, such as granular media, emulsions, wet foams, weakly connected biopolymer networks and covalent network glasses [6-8] can all be prepared in a fragile state. Second, all of these materials exhibit a vanishing range of linear response in their fragile state. As a consequence, tiny perturbations can elicit extreme mechanical responses in the form of shocks, rearrangements, yielding and flow [6-10]. It is tempting to ask whether, similar to shock waves in granular packings, all of these complex phenomena can be described by simple scaling relations. -

\section{About the Authors}

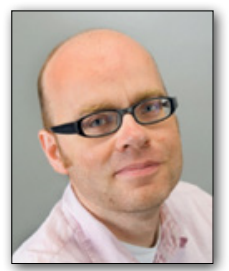

Martin van Hecke leads an experimental/numerical group at the university of Leiden, the Netherlands. His research interest is the mechanics and organization of disordered media, such a granular matter, foams, and elastic networks.

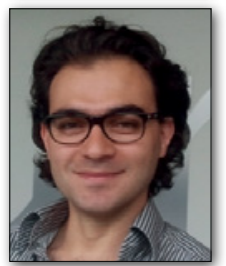

Vincenzo Vitelli leads a theoretical group in the Institute Lorentz, Leiden University. His interests are in soft condensed matter theory and statistical mechanics.

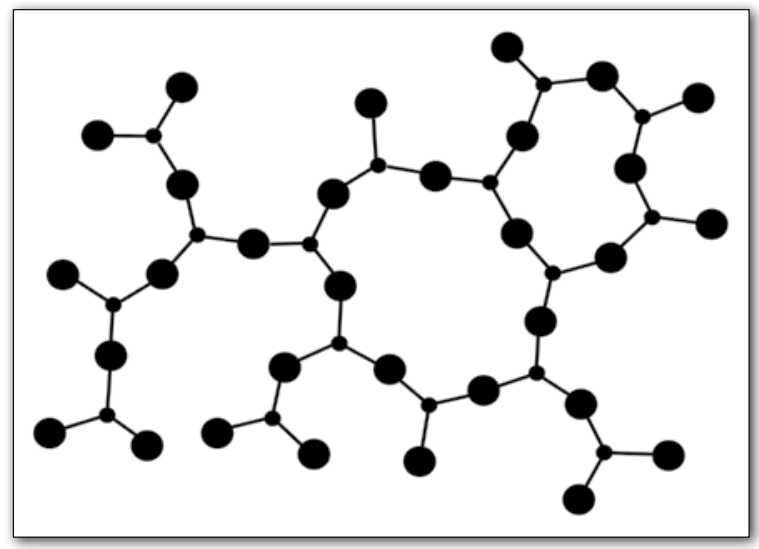

4 Fig. 5: Network glasses such as chalcogenides, illustrated here, can become fragile too.

\section{References}

[1] V.F. Nesterenko, J. Appl. Mech. Tech. Phys. 5, 733 (1984); and Dynamics of Heterogeneous Materials (Springer-Verlag, New York, 2001).

[2] A. Spadoni and C. Daraio, Proceedings of the National Academy of Sciences 107, 7230 (2010); N. Boechler, G. Theocharis and C. Daraio, Nature Materials 107, 7230 (2010).

[3] X. Jia, C. Caroli, B. Velicky, Phys. Rev. Lett. 82, 1863 (1999).

[4] L. R. Gomez, A. Turner, M. van Hecke, V. Vitelli, Phys. Rev. Lett. 108, 058001 (2012).

[5] J. C. Phillips, J. Non-Cryst. Solids 43, 37 (1981); M.F. J. Thorpe, Non-Cryst. Solids 57, 355 (1983).

[6] M. Wyart, H. Liang, A. Kabla, and L. Mahadevan, Phys. Rev. Lett. 101, 215501 (2008); C. P. Broedersz, X. Mao, T. C. Lubensky, and F. C. MacKintosh, Nature Physics 7, 983 (2011).

[7] P. Olsson and S. Teitel, Phys. Rev. Lett. 99, 178001 (2007); B.P.Tighe, E. Woldhuis, J.J.C. Remmers, W. van Saarloos, and M. van Hecke, Phys. Rev. Lett. 105, 088303 (2010).

[8] M. van Hecke, J. Phys.: Condens. Matter 22, 033101 (2010).

[9] N. Xu, V. Vitelli, M. Wyart, A.J. Liu, and S.R. Nagel, Phys. Rev. Lett. 102, 038001 (2009).

[10] K.N. Nordstrom, E. Verneuil, P.E. Arratia, A. Basu, Z. Zhang, A.G. Yodh, J.P. Gollub, and D.J. Durian, Phys. Rev. Lett. 105, 175701 (2010).

\section{BOX 2: SCALING}

If a granular medium is compressed, once transients have died out, the front propagates with constant speed $v_{s}$ whose dependence on piston speed $u_{p}$ is illustrated in Fig. 4 for different values of the applied pressure $P$ [4]. Here, both $v_{s}$ and $u_{\mathrm{p}}$ are measured in units of the speed of sound $v_{\mathrm{g}}$ in the individual grain. For low $u_{\mathrm{p}}$, the front speed $v_{\mathrm{s}}$ is nearly independent of $u_{p}$ - in this (quasi)linear regime, $v_{s}$ is simply controlled by the initial pressure $P$. In contrast, for high impact intensity, the nonlinear shock wave regime is reached, where $v_{s} \sim u_{p}^{1 / 5}$, but does not depend on $P$. The physical origin of the shocks can be grasped by means of intuitive arguments and scaling. Even if the grains are not in touch to start with, the propagating front itself will generate a compression $\delta$ and a corresponding increase in the kinetic energy. Combining mass conservation through the shock with the assumption that the kinetic and potential energies are of the same order in the regime behind the shock, one obtains a scaling relation for the shock speed; $v_{\mathrm{s}} \sim u_{\mathrm{p}}{ }^{1 / 5}$. This scaling relation, plotted as a dashed line in Fig. 4, is clearly consistent with the numerical data.

FIG. 4: Scaling plot revealing the relations between the shock speed $v_{s}$ piston speed $u_{p}$, overlap $\delta$ and sound speed within the material, $\mathbf{v}_{\mathrm{g}}$.

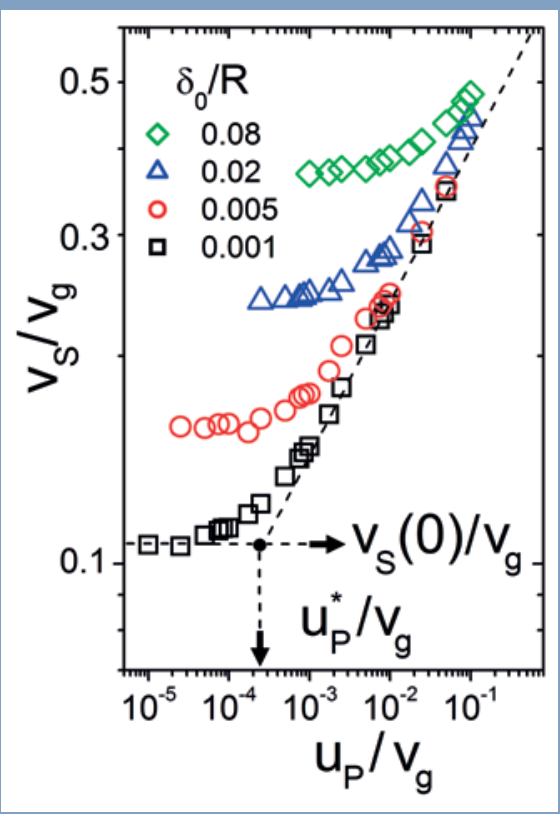

